\title{
Escolha de cultivares de soja com base na composição química dos grãos como perspectiva para maximização dos lucros nas indústrias processadoras
}

\author{
The selection of soybean varieties based on the chemical composition of the grains as a \\ mean of maximizing soybean processing industry's profits
}

\author{
Adriana Sbardelotto ${ }^{\mathrm{I}}$ Gideon Villar Leandro ${ }^{\mathrm{II}}$
}

\section{RESUMO}

Apresenta-se um modelo matemático baseado em programação linear para dar suporte às decisões referentes à escolha de cultivares de soja para processamento (extração de óleo e produção de farelo), de forma que esta escolha possa maximizar os lucros da indústria processadora. Este estudo utilizou amostras de nove cultivares de soja produzidas no município de Dois Vizinhos, região sudoeste do Estado do Paraná. Análises laboratoriais forneceram as respectivas composições dos grãos, os subprodutos, os resíduos e as perdas. O modelo permitiu estimar os retornos econômicos proporcionados à indústria processadora por meio do cultivo individual e pela média destas. A partir da análise dos resultados, conclui-se que a composição química dos grãos processados tem influência direta nos resultados econômicos da indústria e que o esmagamento das cultivares "BRS133”, “CD215”, “EMBRAPA48”, “BRS184”, "SPRING8350” $e$ “MSOY5826" pode maximizar os lucros da indústria processadora, enquanto que o esmagamento das cultivares “CD 205”, “CD 206” e "BRS 214” pode reduzir os lucros, em relação à média obtida pelas cultivares avaliadas.

Palavras-chave: programação linear, modelo matemático, cultivares de soja.

\section{ABSTRACT}

This article presents a mathematical model based on linear programming to support decision-making when selecting the soybean variety to be processed for oil extraction and bran production, since the appropriate choice can maximize industry's profits. Samples of nine soybean varieties produced in the town of Dois Vizinhos, in the southwest region of the state of Paraná, Brazil, were used in this study. Laboratory analyses revealed the chemical composition of the grains, the byproducts, residues and losses. The model made it possible to estimate the profits from the processing of each variety and of the nine varieties' average. Our results show that the chemical composition of the grains directly influences the industry's profits and that the processing of the varieties 'BRS133', 'CD215', 'EMBRAPA48', 'BRS184', 'SPRING8350' and 'M-SOY5826' can maximize the profits, while the processing of the varieties ' $C D$ 205', ' $C D$ 206' and 'BRS 214' can reduce them, compared to the average obtained from the investigated varieties.

Key words: linear programming, mathematical model, soybean variety.

\section{INTRODUÇÃO}

A soja é um dos produtos agrícolas mais comercializados no mundo. Isso provavelmente se deve a suas diversas formas de consumo, que se estendem desde a alimentação (humana e animal) até a indústria farmacêutica e siderúrgica. Essa diversidade é possível porque as indústrias de processamento de soja produzem subprodutos (farelo e óleo) que se constituem em importante matéria-prima para diversos setores industriais (FREITAS et al., 2001). O Brasil, segundo maior produtor de grãos de soja do mundo, produziu na safra 2005/06 o montante de 53.053 (1.000 toneladas) de grãos de soja, sendo que destes exportou 22.389 (1.000 toneladas) de grãos, 13.889 (1.000 toneladas) de farelo e 2.595 (1.000 toneladas) de óleo (ABIOVE, 2006).

Com velocidade semelhante à expansão da cultura da soja pelo país, tanto em áreas plantadas

IDepartamento de Física, Estatística e Matemática (DEFEM), Universidade Regional do Noroeste do Estado do Rio Grande do Sul (UNIJUÍ), Ijuí, RS. E-mail: adriana_sbardelotto@yahoo.com.br. Endereço para correspondência: São Valentim, CP 211, Dois Vizinhos, PR, Brasil.

IIDepartamento de Tecnologia, UNIJUÍ, Ijuí, Rio Grande do Sul, Brasil. 
quanto em produtividade, foram desenvolvidas muitas cultivares com características específicas e composição diferenciada de grãos. Essas características compreendem a adaptação ao solo e ao clima, a produtividade, a resistência a doenças e pragas, a resistência à estiagem e as diferenças quanto a ciclo e hábito (SEDYAMA et al., 1999). Já as diferenças na composição dos grãos correspondem aos percentuais de óleo, proteínas, umidade, carboidratos, fibras e cinzas (DEMBORGUSKI, 2003).

As indústrias esmagadoras, as quais movimentam um dos setores mais produtivos da economia brasileira, que é a soja e seu complexo, composto por óleo, farelo e casca (VICENTE et al., 2001), compram a soja pelo peso e pela análise dos fatores relacionados à qualidade dos grãos: umidade, impurezas e avaria. Portanto, a composição química dos grãos das diferentes cultivares em termos de umidade, óleo, proteínas, carboidratos, cinzas e fibras não é considerada.

No processo de esmagamento para obtenção dos subprodutos óleo, farelo e cascas, a soja proveniente de diferentes cultivares se mistura e a composição da massa de grãos esmagada torna-se uma combinação da composição da mistura dessas cultivares.

Neste trabalho, procura-se enfatizar a importância de se analisar a composição química dos grãos, já que existem diferenças na composição entre as cultivares de soja. Além disso, busca-se mostrar com um modelo de programação linear que a escolha adequada das cultivares implicará na maximização dos lucros da indústria processadora, uma vez que os custos da saca da soja, da limpeza e da secagem, do armazenamento, do processo de extração de óleo e da produção do farelo são os mesmos para qualquer cultivar, desde que estas estejam no mesmo padrão de umidade e qualidade.
Os modelos matemáticos servem de auxílio à decisão em geral, pois retratam o sistema e procuram determinar qual é a combinação de fatores que atende ao objetivo estabelecido, que pode ser minimizar os custos ou maximizar os lucros. Um problema descrito por um modelo pode ser resolvido por Programação Linear (PL) se as variáveis são contínuas e se a função objetivo e todas as restrições são descritas por funções lineares. Uma vez obtido o modelo linear, constituído pela função objetivo e pelas restrições, a programação linear se incumbe de obter pelo menos uma solução ótima para o problema, se ela existir (GOLBARG \& LUNA, 2000; ANDRADE, 2000).

Com este trabalho, propõe-se uma nova perspectiva para a indústria ampliar seus lucros, por meio de uma alternativa que possibilita a maximização da receita das indústrias processadoras de soja. A maximização ocorre a partir da escolha ótima de cultivares de soja, com base em resultados de análises das respectivas composições químicas dos grãos.

\section{MATERIAL E MÉTODOS}

A composição da massa de grãos apresentada por DEMBORGUSKI (2003) (Tabela 1) é construída a partir da composição dos grãos de soja (valores médios) e de seus subprodutos, além dos resíduos e da estimativa de perdas. Os dados são provenientes de análises laboratoriais realizadas em cinco indústrias extratoras de óleo da região noroeste do Estado do Rio Grande do Sul.

Para se obter os dados das diversas cultivares de soja plantadas no município de Dois Vizinhos, na safra 2005/06, foi realizada uma pesquisa junto às principais revendedoras de sementes e insumos, a fim de coletar os dados sobre o nome das cultivares vendidas e as respectivas quantidades. Nesta pesquisa, constatou-se que foram nove as

Tabela 1 - Percentuais relativos dos componentes na composição do grão de soja, de seus subprodutos, resíduos e estimativa da composição das perdas.

\begin{tabular}{|c|c|c|c|c|c|c|c|}
\hline Composição relativa (\%) & Grão & Óleo & Farelo & Casca & Resíduo & Perda & Lecitina $^{\text {III }}$ \\
\hline Umidade(1) & 12,5 & 0,15 & 12,5 & 7,25 & 11 & 60 & 0 \\
\hline Óleo(2) & 19,5 & 99,7 & 2 & 0,77 & 0,9 & 10 & 24,2 \\
\hline Proteínas(3) & 36,6 & 0 & 47 & 8,88 & 20 & 10 & 0 \\
\hline Fibras(4) & 5,5 & 0 & 3,92 & 34,88 & 0 & 10 & 0 \\
\hline Impurezas(5) & 0,2 & 0 & 0 & 0 & 30 & 10 & 0 \\
\hline Fosfato(6) & 1,0 & 0,15 & 0,8 & 0,72 & 0 & 0 & 75,8 \\
\hline Carboidratos(7) & 24,7 & 0 & 33,78 & 51,78 & 20 & 0 & 0 \\
\hline Produção final(8) & $100 \%$ & $18,13 \%$ & $70,74 \%$ & $2,81 \%$ & 0 & $7,84 \%$ & $0,48 \%$ \\
\hline
\end{tabular}

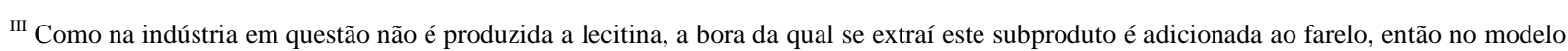
soma-se o percentual do subproduto lecitina com percentual do subproduto farelo.

Fonte: DEMBORGUSKI (2003).

Ciência Rural, v.38, n.3, mai-jun, 2008. 
cultivares mais plantadas na safra 2005/06. Ordenandoas decrescentemente da mais plantada para as menos plantadas, tem-se a seguinte relação: “CD206”, “CD215”, “Embrapa48”, “BRS184”, “M-SOY5826”, “CD205”, “BRS214”, “Spring NK8350”, “BRS133”.

Então foram adquiridas amostras destas cultivares junto a uma sementeira da região. Essas amostras eram compostas por sementes que ainda não haviam sido tratadas para o plantio. Depois elas foram submetidas a análises físico-químicas para se determinar as composições do grão de cada cultivar com relação aos teores de óleo (método de Soxlet), proteínas (método de Kjeldhal), fibras (método enzímicogravimétrico), carboidratos (método do reagente de Fehling), cinzas (método gravimétrico) e umidade (método da estufa a vácuo).

Foram realizadas visitas a indústrias esmagadoras de soja da região, onde se obteve conhecimento do processo de extração do óleo de soja, da receita e dos custos desse processo. Finalmente, foi elaborado um modelo de programação matemática, a partir do ferramental da PL, a fim de se buscar a maximização dos lucros da indústria. Para resolver o modelo de PL proposto, utilizou-se os softwares Matlab 6.0 e Lingo 7.0. O modelo desenvolvido tem por objetivo otimizar o sistema produtivo da indústria processadora, e os resultados obtidos encontram uma solução ótima para cada cultivar.

O modelo foi aplicado para os dados provenientes de cada uma das nove cultivares individualmente e para a média, considerando-se igual quantidade de grãos de cada cultivar, obtendo-se assim as estimativas dos respectivos lucros.

A forma padrão, utilizada pelos métodos de Programação Linear, é:

$\mathrm{Z}=\sum_{j=1}^{n} c_{j} X_{j}$, neste trabalho o objetivo é maximizar $\mathrm{Z}$ sujeito às restrições:

$$
\sum_{i=1}^{m} \sum_{j=1}^{n} a_{i j} x_{j}=b_{i} \quad,\left(\text { ou } \leq \mathrm{b}_{\mathrm{i}}, \text { ou } \geq \mathrm{b}_{\mathrm{i}} \text {, ou }<\mathrm{b}_{\mathrm{i}} \text { ou }>\mathrm{b}_{\mathrm{i}}\right) \text { com }
$$

$0 \leq x_{j} \leq \mathrm{d}_{j}$

Sendo que:

$\mathrm{Z}$ = função objetivo que corresponde ao resultado econômico da indústria processadora de soja;

$c_{j}=$ coeficiente de lucro para a variável $x_{j}$, isto é, a receita proveniente da venda dos respectivos subprodutos (farelo, óleo e cascas), subtraída dos custos referentes ao sistema de processamento da soja;

$x_{j}=$ variável de decisão, que compõe a quantidade produzida dos subprodutos: farelo, óleo e cascas; $a_{i j}=$ coeficiente da variável $x_{j}$ na restrição $\mathrm{i}$, que indica o peso dos nutrientes (umidade, óleo, proteínas, fibras, cinzas e carboidratos) na composição dos grãos, dos subprodutos, dos resíduos e das perdas;

$b_{i}=$ valor limite da restrição i, que indica os valores máximos ou mínimos a serem atingidos por cada nutriente ou subproduto nas respectivas restrições. $d_{j}$ = quantidade máxima da variável $x_{j}$;

$\mathrm{i}=1,2, \ldots, \mathrm{m}(\mathrm{m}=23)$ - número de restrições ou condições impostas;

$\mathrm{j}=1,2, \ldots, n(n=15)$ - número de variáveis.

O modelo utilizado neste trabalho foi proposto por DEMBORGUSKI (2003), com adaptações referentes à composição do grão, dos subprodutos e das perdas. Aqui são utilizados os valores referentes à composição de cada uma das nove cultivares e ainda uma composição formada pela média aritmética das nove. Os valores limites referentes aos subprodutos são adequados a cada cultivar, sendo que os valores das composições das cultivares são aqueles obtidos através das análises laboratoriais cujos resultados são apresentados na tabela 2 .

No modelo proposto, são considerados sete grupos de restrições relacionadas à composição dos grãos (disponibilidade de umidade, óleo, proteínas, fibras, impurezas, cinzas e carboidratos) e três grupos de restrições relacionadas aos subprodutos da soja (óleo, farelo e casca), elaboradas a partir dos dados apresentados na tabela 1. A estrutura da função objetivo e das restrições é a seguinte.

\section{Função objetivo}

Poleo $\left(x_{i} O_{1}+x_{i} O_{2}+x_{i} O_{6}\right)+$ Pfarelo $\left(x_{i} f_{1}+x_{i}\right.$ $\left.f_{2}+x_{i} f_{3}+x_{i} f_{4}+x_{i} f_{6}+x_{i} f_{7}\right)+$ Pcasca $\left(x_{i} c_{1}+x_{i} c_{2}+x_{i}\right.$ $\left.c_{3}+x_{i} c_{4}+x_{i} c_{6}+x_{i} c_{7}\right)-$ Custos

Para i $=1,2, \ldots, 10$. Onde cada valor de $\mathrm{i}=1$, $2, \ldots, 9$ representa uma cultivar, e $\mathrm{i}=10$ representa a composição média das nove cultivares.

Os coeficientes da função objetivo são: Poleo - preço de venda por kg de óleo degomado, Pfarelo - preço de venda por kg de farelo, Pcasca preço de venda por kg de casca, Custos - são referentes à: uma saca de soja $(60 \mathrm{~kg})+$ limpeza + secagem + armazenamento + processo de extração.

As variáveis que serão determinadas pelo modelo são:

$x_{i} O_{1}$ - umidade no óleo, $x_{i} O_{2}$ - óleo no óleo, $x_{i} O_{6}$ - cinzas no óleo, $x_{i} f_{1}$ - umidade no farelo, $x_{i} f_{2}$ - óleo no farelo, $x_{i} f_{3}$ - proteínas no farelo, $x_{i} f_{4}$ - fibras no farelo, $x_{i} f_{6}$ cinzas no farelo, $x_{i} f_{7}$-carboidratos no farelo, $x_{i} c_{1}$ umidade na casca, $x_{i} C_{2}$ - óleo na casca, $x_{i} C_{3}$ - proteínas

Ciência Rural, v.38, n.3, mai-jun, 2008. 
Tabela 2 - Composição dos grãos das cultivares de soja analisadas e a composição média.

\begin{tabular}{|c|c|c|c|c|c|c|c|c|c|c|}
\hline Composição relativa(\%) & $\begin{array}{l}\text { “CD } \\
205 ”\end{array}$ & $\begin{array}{l}\text { “CD } \\
206 ”\end{array}$ & “C215” & $\begin{array}{l}\text { “Spring } \\
\text { 8350”" }\end{array}$ & $\begin{array}{l}\text { “Msoy } \\
5826 ”\end{array}$ & $\begin{array}{c}\text { “Embrapa } \\
48 ”\end{array}$ & $\begin{array}{l}\text { “BRS } \\
133 ”\end{array}$ & $\begin{array}{l}\text { "BRS } \\
184 "\end{array}$ & $\begin{array}{l}\text { “BRS } \\
214 ”\end{array}$ & $\begin{array}{l}\text { Composição } \\
\text { média }^{2}\end{array}$ \\
\hline Umidade $(1)^{1}$ & 10,6 & 11,2 & 10,2 & 11,3 & 10 & 10,8 & 10 & 10,6 & 11,2 & 10,7 \\
\hline Óleo(2) & 20,9 & 21,4 & 22,5 & 22,6 & 22,7 & 22,1 & 22,9 & 22,5 & 21 & 22,1 \\
\hline Proteínas(3) & 37,5 & 38,4 & 38 & 37,5 & 37,8 & 37,1 & 36,6 & 37,7 & 38,2 & 37,6 \\
\hline Fibras(4) & 4 & 3,8 & 3,1 & 3,4 & 3,8 & 4 & 3,6 & 3,8 & 4,2 & 3,7 \\
\hline Impurezas(5) & 0 & 0 & 0 & 0 & 0 & 0 & 0 & 0 & 0 & 0 \\
\hline Cinzas(6) & 5,1 & 4,2 & 4,7 & 4,3 & 4,9 & 4,5 & 4,5 & 4,4 & 4,6 & 4,6 \\
\hline Carboidratos (7) & 21,9 & 21 & 21,5 & 20,9 & 20,8 & 21,5 & 22,4 & 21 & 20,8 & 21,3 \\
\hline
\end{tabular}

${ }^{1}$ Na composição média considera-se igual quantidade de grãos de cada cultivar.

${ }^{2}$ Os índices (1), (2), ...., (7) estão diretamente ligados a variáveis do modelo, uma vez que a tabela 2 fornece os valores limites para as restrições do modelo, por exemplo, o óleo produzido é composto por umidade, óleo e cinzas $\left(X_{i} O_{1}+X_{i} O_{2}+X_{i} O_{6}\right)$, em que os índices 1 , 2 e 6 estão definindo em quais linhas estão os componentes do óleo.

na casca, $x_{i} C_{4}$ - fibras na casca, $x_{i} C_{6}$ - cinzas na casca, $x_{i} c_{7}$ - carboidratos na casca, $x_{i} r_{1}$ - umidade no resíduo, $x_{i} r_{2}$ - óleo no resíduo, $x_{i} r_{3}$ - proteínas no resíduo, $x_{i} r_{5}$ impurezas no resíduo, $x_{i} r_{7}$ - carboidratos no resíduo, $x_{i} p_{1}$ - perda de umidade, $x_{i} p_{2}$ - perda de óleo, $x_{i} p_{3}$ perda de proteínas, $x_{i} p_{4}$ - perda de cinzas, $x_{i} p_{5}$ - perda de impurezas, $x_{i} l_{2}$ - óleo na lecitina, $x_{i} l_{6}$ - cinza na lecitina.

Os valores limites das restrições são: $b_{i 1}$ quantidade máxima de umidade no grão, $b_{i 2}$ - quantidade máxima deóleo no grão, $b_{i 3}$-quantidademáxima deproteínas no grão, $b_{i 4}$ - quantidade máxima de fibras no grão, $b_{i 5}$ quantidade máxima de impurezas no grão, $b_{i 6}$ - quantidade máxima de impurezas no grão, $b_{i 7}$ - quantidade máxima de carboidratos no grão.

a) Restrições associadas à composição do grão.

Restrição da disponibilidade de umidade

$x_{i} O_{1}+x_{i} f_{1}+x_{i} C_{1}+x_{i} r_{1}+x_{i} p_{1} \leq b_{i 1}$

Restrição da disponibilidade de óleo

$x_{i} O_{2}+x_{i} f_{2}+x_{i} C_{2}+x_{i} r_{2}+x_{i} p_{2}+x_{i} l_{2} \leq b_{i 2}$

Restrição da disponibilidade de proteínas

$x_{i} f_{3}+x_{i} c_{3}+x_{i} r_{3}+x_{i} p_{3} \leq b_{i 3}$

Restrição da disponibilidade de fibras

$x_{i} f_{4}+x_{i} c_{4}+x_{i} p_{4} \leq b_{i 4}$

Restrição da disponibilidade de impurezas

$x_{i} r_{5}+x_{i} p_{5} \leq b_{i 5}$

Restrição da disponibilidade de cinzas

$x_{i} O_{6}+x_{i} f_{6}+x_{i} c_{6}+x_{i} l_{6} \leq b_{i 6}$

Restrição da disponibilidade de carboidratos

$x_{i} f_{7}+x_{i} C_{7}+x_{i} r_{7} \leq b_{i 7}$

b) Restrições associadas aos subprodutos da soja.

Restrições para o óleo

$x_{i} O_{1} \leq 0,0015\left(x_{i} O_{1}+x_{i} O_{2}+x_{i} O_{6}\right)$

$x_{i} O_{2} \leq 0,997\left(x_{i} O_{1}+x_{i} O_{2}+x_{i} O_{6}\right)$ $x_{i} O_{6} \leq 0,0015\left(x_{i} O_{1}+x_{i} O_{2}+x_{i} o_{6}\right)$

$x_{i} O_{1}+x_{i} O_{2}+x_{i} O_{6} \leq\left(b_{i 2} * 0,915\right)$

Restrições para o farelo

$x_{i} f_{1} \leq 0,125\left(x_{i} f_{1}+x_{i} f_{2}+x_{i} f_{3}+\right.$ $\left.x_{i} f_{4}+x_{i} f_{6}+x_{i} f_{7}\right)$

$x_{i} f_{2} \leq 0,02\left(x_{i} f_{1}+x_{i} f_{2}+x_{i} f_{3}+x_{i} f_{4}+x_{i}\right.$ $\left.f_{6}+x_{i} f_{7}\right)$

$x_{i} f_{3} \leq 0,46\left(x_{i} f_{1}+x_{i} f_{2}+x_{i} f_{3}+x_{i} f_{4}+x_{i}\right.$ $\left.f_{6}+x_{i} f_{7}\right)$

$x_{i} f_{3} \leq 0,47 \quad\left(x_{i} f_{1}+x_{i} f_{2}+x_{i} f_{3}+\right.$

$\left.x_{i} f_{4}+x_{i} f_{6}+x_{i} f_{7}\right)$

$x_{i} f_{4} \leq 0,0392\left(x_{i} f_{1}+x_{i} f_{2}+x_{i} f_{3}+\right.$

$\left.x_{i} f_{4}+x_{i} f_{6}+x_{i} f_{7}\right)$

$x_{i} f_{6} \leq 0,008\left(x_{i} f_{1}+x_{i} f_{2}+x_{i} f_{3}+\right.$

$\left.x_{i} f_{4}+x_{i} f_{6}+x_{i} f_{7}\right)$

$x_{i} f_{7} \leq 0,3378\left(x_{i} f_{1}+x_{i} f_{2}+x_{i} f_{3}+\right.$ $\left.x_{i} f_{4}+x_{i} f_{6}+x_{i} f_{7}\right)$

$x_{i} f_{1}+x_{i} f_{2}+x_{i} f_{3}+x_{i} f_{4}+x_{i} f_{6}+x_{i} f_{7} \leq$ $\left(100-b_{i 2} * 0,915-2,81-7,84\right)$

Restrições para a casca

$x_{i} c_{1} \leq 0,0725 \quad\left(x_{i} c_{1}+x_{i} c_{2}+x_{i} c_{3}+\right.$ $\left.x_{i} C_{4}+x_{i} C_{6}+x_{i} C_{7}\right)$

$x_{i} c_{2} \leq 0,0077 \quad\left(x_{i} c_{1}+x_{i} c_{2}+x_{i} c_{3}+\right.$ $\left.x_{i} C_{4}+x_{i} C_{6}+x_{i} C_{7}\right)$

Ciência Rural, v.38, n.3, mai-jun, 2008. 
$x_{i} c_{3} \leq 0,0888 \quad\left(x_{i} c_{1}+x_{i} c_{2}+x_{i} c_{3}+\right.$

$\left.x_{i} C_{4}+x_{i} C_{6}+x_{i} C_{7}\right)$

$x_{i} c_{4} \leq 0,3488 \quad\left(x_{i} c_{1}+x_{i} c_{2}+x_{i} c_{3}+\right.$

$\left.x_{i} C_{4}+x_{i} C_{6}+x_{i} C_{7}\right)$

$x_{i} \quad c_{6} \leq 0,0072 \quad\left(x_{i} c_{1}+x_{i} c_{2}+x_{i} c_{3}+\right.$

$\left.x_{i} c_{4}+x_{i} c_{6}+x_{i} c_{7}\right)$

$x_{i} \quad c_{7} \leq 0,0888 \quad\left(x_{i} c_{1}+x_{i} c_{2}+x_{i} c_{3}+\right.$

$\left.x_{i} C_{4}+x_{i} C_{6}+x_{i} C_{7}\right)$

$x_{i} C_{1}+x_{i} C_{2}+x_{i} C_{3}+x_{i} c_{4}+x_{i} c_{6}+x_{i} c_{7} \leq$ 2,81

\section{RESULTADOS E DISCUSSÕES}

Na tabela 3, são apresentados os resultados referentes à função objetivo que denota o lucro da indústria processadora ao esmagar uma tonelada de cada cultivar de soja. Também é apresentado o lucro diário proveniente do esmagamento de 470 toneladas. O lucro obtido com a composição média das cultivares é considerado como o mais próximo dos valores reais da indústria, por isso o lucro médio foi utilizado como valor de referência. Há diferenças na estimativa de lucro entre as cultivares de soja, sendo que para seis cultivares estimou-se maiores retornos que a média e para as outras três estimou-se menores retornos do que o lucro médio.

Os resultados obtidos com o modelo proposto têm como referencial uma planta industrial localizada no município de Dois Vizinhos (sudoeste do Estado do Paraná), principal comprador da soja

Tabela3 - Lucros obtidos (em R\$) no processamento das cultivares de soja analisadas.

\begin{tabular}{lcc}
\hline Cultivares & Lucro/t (R\$) & Lucro/dia (R\$) \\
\hline “CD 205” & 705,38 & $331.528,60$ \\
“CD 206” & 698,61 & $328.346,70$ \\
“CD 215” & 725,15 & $340.820,50$ \\
“SPRING 8350” & 716,73 & $336.863,10$ \\
“M-SOY 5826” & 716,68 & $336.839,60$ \\
“EMBRAPA 48” & 718,49 & $337.690,30$ \\
“BRS 133” & 746,68 & $350.939,60$ \\
“BRS 184” & 716,79 & $336.891,30$ \\
“BRS 214” & 688,78 & $323.726,60$ \\
Média & 715,15 & $336.120,50$ \\
\hline
\end{tabular}

Fonte: Dados da pesquisa. produzida na região, entretanto a idéia proposta pode ser aplicada a outras indústrias processadoras.

A tabela 4 apresenta um resumo das diferenças entre o lucro médio e os lucros resultantes de cada cultivar. Ao observá-la, percebe-se que as cultivares "BRS133”, “CD215”, "EMBRAPA48”, “BRS184”, “SPRING8350” e “M-SOY5826” podem aumentar significativamente os lucros da indústria processadora. Estes aumentos acumulados no decorrer do ano poderiam gerar um retorno extra de R\$ 5.334.876,00, R\$ 1.692.000,00, R\$ 565.128,00, R\$ $277.488,00$, R\$ 267.336,00 e R\$258.876,00 respectivamente. Entretanto, outras cultivares como “BRS214”, “CD206” e “CD205” podem causar uma redução anual de $\mathrm{R}$ \$ 4.461.804,00, R \$ 2.798.568,00 e R \$ 1.653.084,00, respectivamente.

Ao correlacionar a composição das cultivares (Tabela 2) e o retorno financeiro gerado por estas à indústria processadora, percebe-se que existe uma relação direta entre a soma dos percentuais de óleo e carboidratos com o lucro da indústria. A justificativa dessa relação reside no fato de que o subproduto óleo agrega maior valor que os demais e os carboidratos constituem o segundo maior componente do farelo. As proteínas, embora constituam o maior componente presente no farelo, cinzas, fibras e umidade não demonstram relação direta com os lucros.

Observando estes resultados econômicos da indústria no processamento de tais cultivares de soja, notam-se diferenças relevantes, que poderiam ser usadas estrategicamente pela indústria como alternativa para a maximização dos lucros. Os modelos de programação linear baseados em dados experimentais são instrumentos muito utilizados em análises econômicas no campo agroindustrial de países desenvolvidos (GIBBIN, 2005). Considerando que a realidade das indústrias processadoras consiste na compra de várias cultivares em diferentes proporções, isso condiz com a metodologia proposta neste trabalho (composição média) e confirma a funcionalidade prática do mesmo.

Com estas informações, a indústria processadora poderá planejar em conjunto com os produtores quais as cultivares serão plantadas. Isso estimula que os produtores plantem as cultivares que lhe proporcionam maiores retornos financeiros, uma vez que a indústria obtendo maior lucro possibilita pagar um valor diferenciado na compra das sojas. Isso qualificará o planejamento da produção, deixando a indústria menos suscetível a incertezas quantos aos resultados esperados.

Ciência Rural, v.38, n.3, mai-jun, 2008. 
Tabela 4 - Comparações entre o lucro de cada cultivar de soja e o lucro médio ${ }^{1}$.

\begin{tabular}{lcc}
\hline Cultivares & Lucro por cultivar - lucro médio (t) & Percentual da diferença com relação ao lucro médio \\
\hline “CD 205” & $-\mathrm{R} \$$ 9,77 & $-1,37 \%$ \\
“CD 206” & $-\mathrm{R} \$ 16,54$ & $-2,31 \%$ \\
“CD 215” & $\mathrm{R} \$ 10,00$ & $1,40 \%$ \\
“SPRING 8350” & $\mathrm{R} \$ 1,58$ & $0,22 \%$ \\
"M-SOY 5826” & $\mathrm{R} \$ 1,53$ & $0,21 \%$ \\
“EMBRAPA 48” & $\mathrm{R} \$ 3,34$ & $0,47 \%$ \\
“BRS 133” & $\mathrm{R} \$ 31,53$ & $4,41 \%$ \\
“BRS 184” & $\mathrm{R} \$ 1,64$ & $0,23 \%$ \\
"BRS 214” & $-\mathrm{R} \$ 26,37$ & $-3,69 \%$ \\
\hline
\end{tabular}

${ }^{1}$ O lucro médio é usado como ponto de referência, pois este representa o lucro proporcionado à indústria pelo esmagamento da mistura de grãos que possui a composição média, já referenciada anteriormente.

\section{CONCLUSÕES}

As diferenças na composição química dos grãos das cultivares de soja interferem no retorno financeiro da indústria processadora. Portanto, sugerese que estas passem a analisar a composição da soja comprada. Dentre as nove cultivares que foram analisadas neste trabalho, estimou-se que seis delas (“BRS133”, “CD215”, “EMBRAPA48”, "BRS184”, “SPRING8350” " “M-SOY5826”) propiciariam aumentos em relação ao lucro médio.

\section{REFERÊNCIAS}

ABIOVE - Associação Brasileira das Indústrias de Óleos Vegetais Estatísticas do Complexo Soja. Acesso em 12 de nov. 2006. On line. Disponível na internet: http://www.abiove.com.br/abiov.

ANDRADE, E.L. Introdução à pesquisa operacional: métodos e modelos para a análise de decisão. 2.ed. Rio de Janeiro: LTC, 2000. 277p.
DEMBOGURSKI, N.M.S.S. Determinação do preço da soja para trituração e obtenção do óleo com base na qualidade do grão. 2003. 86f. Dissertação (Mestrado em Modelagem Matemática) - Universidade Regional do Noroeste do Estado do Rio Grande do Sul, Ijuí.

FREITAS, S.M. et al. Análise da dinâmica de transmissão de preços no Mercado Internacional de Farelo de Soja, 1990-99. Revista Agricultura em São Paulo, v.48, n.1, p.1-20, 2001.

GIBBIN, R.V. Modelos para a escolha de cultivares em empreendimentos agrícolas de pequeno porte. 2005. 82f. Dissertação (Mestrado em Engenharia Mecânica ) Universidade Estadual de Campinas.

GOLBARG, M.C.; LUNA, H.P.L. Otimização combinatória e programação linear - modelos e algoritmos. Rio de Janeiro: Campus, 2000. 649p.

SEDYAMA, T. et al. Melhoramento da soja. In: BORÉM, A. Melhoramento de espécies cultivadas. Viçosa: UFV, 1999. p.487-533.

VICENTE, J.R. et al. Produtividade agrícola no Brasil: 19701995. Revista Agricultura em São Paulo, v.48, n.2, p.3355, 2001. 